\title{
Analyse der Spaltungsprodukte der Gelatine.
}

\author{
Von \\ P. A. Levene und W. A. Beatty. \\ (Aus dem Rockefeller Institute for Medical Research, New York.) \\ (Der Redaktion zugegangen am 3. September 1906.)
}

Die hydrolytischen Spaltungsprodukte der Gelatine sind der Gegenstand so vieler Untersuchungen gewesen, daß eine Wiederholung derselben kaum nötig scheint. Die vorliegende Arbeit wurde unternommen nicht so sehr mit der Absicht, die $\mathrm{Zu}-$ sammensetzung der Gelatine zu erforschen, als um eine Analyse der Spaltungsprodukte auszuarbeiten, bei welcher man die Fischersche Estermethode nicht zu Hilfe zu nehmen braucht. Das Bedürfnis nach einem solchen Verfahren war sehr dringend, seit einer von uns mit der Untersuchung der teilweisen Spaltung dieses Eiweißstoffes beschäftigt war.

Der Gang der Analyse wurde gegründet auf die Eigenschaft der Aminosäuren, mit Phosphorwolframsäure Verbindungen von verschiedener Beschaffenheit zu bilden, auf die Eigenschaft des Glykokolls, ein mäßig lösliches Pikrat zu bilden, und auf die Unterschiede in der Löslichkeit der Kupfersalze der Aminosäuren.

Die Resultate der bisherigen Bemühungen sind nicht ganz ohne Erfolg, obwohl sie nicht vollkommen befriedigend waren. Ein großer Teil des Stickstoffs ging überhaupt verloren. Dieser Verlust wurde dadurch verursacht, daß unnötig viele Fraktionen dargestellt wurden, und daß nicht genügend Sorgfalt beim Auswaschen der verschiedenen Niederschläge geübt war. Doch wird die durch vorliegende Arbeit gewonnene Erfahrung es ermöglichen, in Zukunft die Analyse mit viel besserem Erfolg zu wiederholen. Wir hoffen Gelegenheit zu haben, diese Arbeit auszuführen. 
$400 \mathrm{~g}$ trockene käufliche Gelatine (Goldmarke) wurden in 21 Salzsäure (spez. Gew. 1,20) (die Lösung enthielt $72 \mathrm{~g}$ Stickstoff) und 6 Stunden am Rückflußkühler gekocht, dann unter vermindertem Druck eingedampft, der Rückstand in Wasser aufgelöst, und die Lösung wieder unter vermindertem Druck eingedampft. Diese Operation wurde einigemal wiederholt behufs Entfernung des Überschusses der Salzsäure. Der Rückstand wurde endlich in so viel Wasser aufgelöst, daß die Lösung ein Volumen von $6000 \mathrm{ccm}$ ausmachte. Diese Lösung wurde mit einer 10\% igen Lösung von Phosphorwolframsäure so lange behandelt, bis sich bei weiterer Zugabe kein Niederschlag mehr bildete. Es war $13800 \mathrm{ccm}$ des Reagens, also $1380 \mathrm{~g}$ der Säure erforderlich. Der Niederschlag wurde ohne Stehenlassen auf der Saugpumpe filtriert, mehrmals mit Wasser verrieben und wieder filtriert. Die vereinigten Filtrate wurden von Phosphorwolframsäure befreit, bis zu 11 eingedampft, zum Sieden erhitzt und in die heiße Lösung $200 \mathrm{~g}$ Pikrinsäure eingetragen. Anfangs löste sich die Substanz auf, aber beim Abkühlen erstarrte die Lösung zu einer krystallinischen Masse, sodaß es unmöglich war, sie zu filtrieren. Um dies zu erreichen, wurde die Masse mit 1 l Wasser verrieben. Der Niederschlag bestand hauptsächlich aus Glykokoll; das Filtrat enthielt die anderen Säuren.

\section{A. Pikrinsäureniederschlag.}

Wie erwähnt, wurde die Pikrinsäurebehandlung vorgenommen in einer Lösung von Aminosäuren, die an Baryumchlorid reich war. Man muß hier bemerken, daß Pikrinsäure ${ }^{1}$ ) mit freiem Glykokoll oder mit einer Mischung, in welcher diese Substanz überwiegt, leicht in Verbindung tritt; daß aber mit den gesamten Spaltungsprodukten der Gelatine, wenn diese in freiem Zustande sind, sich dabei eine klebrige Masse bildet, während in Anwesenheit von Chlorbaryum sich ein krystallinisches Pikrat ausscheidet.

1) Levene, The Journal of Biological Chemistry, Vol. I, H. 1, 1905. 
Dieser Niederschlag wurde von Pikrinsäure mit Schwefelsäure und Äther befreit, dabei wird selbstverständlich auch der Baryt entfernt. Die Lösung wurde dann auf übjiche Weise von Schwefelsäure und Salzsäure befreit und eingedampft. Dabei scheidet sich etwas Leucin aus. Das Filtrat von diesem wurde zu ganz kleinem Volumen eingedampft und mit einem Überschuß von Kupferoxyd aufgekocht. Das Filtrat von letzterem erstarrte beim Abkühlen zu einer krystallinischen Masse, die aus Glykokollkupfer bestand. Dieses wurde abfiltriert und zum Filtrate $95 \%$ Alkohol bis zu beginnender Opalescenz zugegeben. Nach 24 stündigem Stehen bei $0^{\circ} \mathrm{C}$. bildete sich ein zweiter Niederschlag.

Das erste Kupfersalz wurde von Kupfer befreit, und die Säure aus sehr verdünntem Alkohol krystallisiert. Die Substanz gab das für Glykokoll typische Pikrat, und das ebenso typische rote Sublimat. Die Analyse der Substanz ergab die folgenden Zahlen:

$0,1757 \mathrm{~g}$ der Substanz gaben bei Verbrennung 0,2155 $\mathrm{g} \mathrm{CO}_{2}$ und $0,1038 \mathrm{~g} \mathrm{H}_{2} \mathrm{O}$.

\begin{tabular}{cr}
\multicolumn{2}{c}{ Für $\mathrm{C}_{2} \mathrm{H}_{5} \mathrm{NO}_{2}$} \\
Berechnet: & Gefunden: \\
$\mathrm{C}=32,00 \%$ & $32,40 \%$ \\
$\mathrm{H}=6,66 \%$ & $6,56 \%$
\end{tabular}

Die Ausbeute betrug $60 \mathrm{~g}$ lufttrockene Substanz.

Das zweite Kupfersalz wurde auch von Kupfer befreit und aus verdünntem Alkohol krystallisiert. Die so erhaltene Substanz schmeckte süß, bildete ein weißes Sublimat, gab keine schwerlösliche Verbindung mit Pikrinsäure und besaß die folgende Zusammensetzung.

$0,1463 \mathrm{~g}$ der Substanz gaben bei Verbrennung $0,2166 \mathrm{~g} \mathrm{CO}_{\mathbf{2}}$ und $0,1037 \mathrm{~g} \mathrm{H}_{2} \mathrm{O}$.

$$
\begin{aligned}
& \text { Für } \mathrm{C}_{3} \mathrm{H}_{7} \mathrm{NO}_{2} \\
& \text { Berechnet: } \\
& \mathrm{C}=40,45 \% \\
& \mathrm{H}=7,87 \%
\end{aligned}
$$

Es lag also Alanin vor, die Ausbeute betrug $9 \mathrm{~g}$. 
Das Filtrat vom zweiten Kupfersalze wurde von Kupfer befreit und mit dem Pikrinsäurefiltrate vereinigt.

\section{B. Pikrinsäurefiltrat.}

Es wurde von Pikrinsäure mittels Äther und Schwefelsäure befreit, und die letztere auf übliche Weise quantitativ entfernt. Beim Eindampfen der Lösung der Säuren scheidet sich Leucin aus. Um die Substanz analysenrein zu erhalten, wurde sie in möglichst wenig konzentrierter Salzsäure aufgelöst, bis zu beginnender Krystallisation eingedampft, abkühlen gelassen, das Hydrochlorat abfiltriert, mit Ammoniak alkalisch gemacht, der überschüssige Ammoniak auf dem Wasserbade entfernt, und das Leucin der Krystallisation überlassen. Aus dem Filtrate der ersten Ausscheidung erhält man eine zweite Krystallisation durch Zugabe von Alkohol. Dieser Niederschlag wurde im Xylolbad getrocknet und analysiert, er besaß die folgende Zusammensetzung:

$0,1378 \mathrm{~g}$ der Substanz gaben bei Verbrennung $0,2787 \mathrm{~g} \mathrm{CO}_{2}$ und $0,1231 \mathrm{~g} \mathrm{H}_{2} \mathrm{O}$.

$$
\begin{array}{cc}
\text { Für } & \mathrm{C}_{6} \mathrm{H}_{13} \mathrm{NO}_{2} \\
\text { Berechnet: } & \text { Gefunden: } \\
\mathrm{C}=54,96 \% & 55,23 \% \\
\mathrm{H}=9,92 \% & 9,92 \%
\end{array}
$$

Die Ausbeute an Leucin betrug $27 \mathrm{~g}$.

\section{Phenylalanin.}

Die Abtrennung des Phenylalanins von anderen Aminosäuren ohne Anwendung der Estermethode wurde von E. Schulze und von $\mathrm{E}$. Schulze und Winterstein ausgeführt. In der vorliegenden Arbeit wurde die Mutterlauge vom Leucin weiter eingedampft, es bildete sich dabei eine Ausscheidung, die das Aussehen des Leucins besaß, welche aber bei der Oxydation mittels Schwefelsäure und Bichromat eine merkliche Entwickelung von Benzaldehyd zum Vorschein brachte. Diese Fraktion wurde abfiltriert und weiter durch fraktionierte Krystallisation gereinigt. Der mittlere Teil wurde in möglichst wenig 5\% iger Schwefelsäure aufgelöst und vorsichtig mit einer Lösung von Phosphor- 
wolframsäure behandelt. Es schied sich dabei eine dicke ölige Masse aus, in welcher beim Stehen ein krystallinischer Niederschlag sich bildete. Diese zwei wurden durch Filtrieren getrennt, die krystallinische Fraktion auf übliche Weise zersetzt, die Lösung zur Trockne eingedampft, und ein Teil des Rückstandes wieder in 5\% iger Schwefelsäure aufgelöst und mit Phosphorwolframsäure behandelt. Es bildete sich ein Niederschlag, der aus perlmutterglänzenden Schuppen bestand, welche, wie es schon Schulze und Winterstein angegeben haben, für das Phosphorwolframat des Phenylalanins typisch ist. Zur Analyse reichte die Menge nicht aus.

In einem zweiten Versuche wurde der auf dieselbe Weise erhaltene Rückstand mit einer Lösung von essigsaurem Kupfer niedergeschlagen und das Kupfersalz analysiert.

$0,1189 \mathrm{~g}$ der Substanz gaben 0,252 $\mathrm{g} \mathrm{CuO}$.

Für $\left(\mathrm{C}_{9} \mathrm{H}_{11} \mathrm{NO}_{2}\right)_{2} \mathrm{Cu}$

\section{Berechnet:}

$\mathrm{Cu}=16,16 \%$
Gefunden:

$16,63 \%$

Es lag also Phenylalanin vor, welches scheinbar von Leucin nicht frei war. Es ist uns wahrscheinlich, daß die Trennung des Phenylalanins von Leucin mit größerem Erfolg durch Überführen in die Äthylester der Fraktion, welche bei der Oxydation eine reichliche Ausbeute an Benzaldehyd erzeugt, ausgeführt werden könnte.

Oxyprolin ${ }^{1}$ ) und Glutaminsäure.

Das Filtrat der Phenylalaninleucinfraktion wurde zu sirupartiger Konsistenz eingedampft, mit Schwefelsäure angesäuert und dann mit einer konzentrierten Lösung von Phosphorwolframsäure (4 Teile der Säure zu 1 Teil Wasser) behandelt. Bei dieser Behandlung bildeten sich Niederschläge von verschiedener Beschaffenheit. Der erste hatte eine schmierige Konsistenz, der zweite war schon mehr körnig, und schließlich der dritte bestand aus schönen weißen Krystallen. Die ersten Fraktionen waren leichter löslich im Überschusse vom Reagens, und dessen Zugabe mußte deshalb mit großer Vorsicht ausgeführt werden.

1) Levene und Beatty, The Journal of Exp. Med., Vol. VIII, S. $463,1906$. 
Wenn die erste Fällung als beendet erkannt wurde, dekantierte man die überstehende Flüssigkeit, setzte die Behandlung mit Phosphorwolframsäure fort, bis die zweite Fällung vollendet war, und so weiter, bis weitere Zugabe von Reagens keine Fällung verursachte. Das Filtrat vom dritten Niederschlage enthielt nur noch $2,0 \mathrm{~g}$ Stickstoff. Also aus $72,0 \mathrm{~g}$ konnten etwa $70 \mathrm{~g}$ auf dem beschriebenen Wege gefällt werden. Jeder der drei Niederschläge wurde erst mit einem Überschusse von konzentrierter Phosphorwolframsäurelösung und nachher mit ganz kleinen Portionen heißem Wasser ausgezogen.

Die Auszüge und die Niederschäge wurden auf übliche Weise von Phosphorwolframsäure befreit.

Aus den Auszügen konnten nur Oxyprolin und Glutaminsäure dargestellt werden. Zur Gewinnung von Oxyprolin wurden die von Phosphorwolframsäure befreiten Lösungen auf ein kleines Volumen eingedampft, Alkohol bis zu beginnender Opalescenz zugegeben, und über Nacht bei Gefriertemperatur stehen gelassen; es schied sich dabei ein Niederschlag aus, der beim Umkrystallisieren das typische Aussehen von Oxyprolin besaß, stark süß schmeckte, bei der Sublimation Pyrrol bildete und die folgende Zusammensetzung besaß.

$0,1540 \mathrm{~g}$ der Substanz gaben bei Verbrennung $0,2573 \mathrm{~g} \mathrm{CO}_{\mathrm{z}}$ und $0,0960 \mathrm{~g} \mathrm{H}_{\mathbf{8}} \mathrm{O}$.

\begin{tabular}{cc}
\multicolumn{2}{c}{ Für $\mathrm{C}_{5} \mathrm{H}_{9} \mathrm{NO}_{3}$} \\
Berechnet: & Gefunden: \\
$\mathrm{C}=45,80 \%$ & $45,56 \%$ \\
$\mathrm{H}=6,87 \%$ & $6,92 \%$
\end{tabular}

Die Mutterlauge von Oxyprolin schmeckte nicht mehr süß, sondern stark sauer. Sie wurde wieder eingedampft und mit Alkohol bis zu starker Opalescenz versetzt. Es bildete sich ein Niederschlag, der einmal umkrystallisiert alle Eigenschaften der Glutaminsäure und die folgende Zusammensetzung besaß.

$0,1588 \mathrm{~g}$ der Substanz gaben bei Verbrennung $0,2344 \mathrm{~g} \mathrm{CO}_{2}$ und $0,0900 \mathrm{~g} \mathrm{H}_{2} \mathrm{O}$.

$$
\begin{array}{cc}
\text { Für } \mathrm{C}_{5} \mathrm{H}_{9} \mathrm{NO}_{4} \\
\text { Berechnet: } & \text { Gefunden: } \\
\mathrm{C}=40,81 \% & 40,25 \% \\
\mathrm{H}=6,12 \% & 6,25 \%
\end{array}
$$

Hoppe-Seyler's Zeitschrift f. physiol. Chemie. XIIX. 
Die Niederschläge wurden alle in Kupfersalze übergeführt, die Lösungen dieser Salze bis zur Trockne eingedampft, und die Rückstände mit absolutem Alkohol ausgekocht. Man erhielt auf diese Weise alkohollösliche Kupfersalze neben unlöslichen. Die unlöslichen wurden aus ganz wenig Wasser umkrystallisiert. Es schied sich dabei das Glykokollkupfer aus; aus der Mutterlauge konnte bei Zugabe von ganz wenig Alkohol ein zweiter Niederschlag, der aus Glykokoll und Alanin bestand, erhalten werden.

Es verblieben also neben den erhaltenen Aminosäuren Mutterlaugen: 1. von den Phosphorwolframsäurefällungen, 2. von Glutaminsäure und endlich 3. von Kupferalanin. Sie zeigten alle keine Neigung zur Krystallisation. Nachdem sie von den Fällungsmitteln befreit waren, wurden die Lösungen vereinigt, bis zur Sirupkonsistenz eingedampft, mit Schwefelsäure angesäuert und mit einer konzentrierten Lösung von Phosphorwolframsäure fraktioniert. Man erhielt wieder ähnliche Fraktionen wie bei der ersten Fällung. Die Fraktionen wurden wieder auf übliche Weise von Phosphorwolframsäure befreit, die erhaltenen Lösungen der Aminosäuren so weit wie möglich eingedampft und mit einer Mischung von Alkohol und Äther ausgekocht. Die Rückstände wurden in derselben Weise wie die Niederschläge der ersten Fällungen behandelt. Man erhielt nur ganz kleine Ausbeuten an Glykokoll und Alanin, jedoch bedeutende an Oxyprolin und Glutaminsäure. Die vereinigten Mutterlaugen wurden dann zu ganz kleinem Volumen eingedampft, in der Kälte mit trockner Salzsäure gesättigt und bei Gefriertemperatur stehen gelassen. Es schied sich dabei Glutaminsäurechlorhydrat aus. Die Mutterlauge wurde dann von Salzsäure befreit. Es war aber unmöglich, aus dieser Fraktion Asparaginsäure zu krystallisieren. Um das zu erreichen, wurde versucht, die nachbleibende Aminosäure durch Erhitzen im Autoklaven mit Barythydrat zu racemisieren. Es schied sich dabei beim Abkühlen ein geringer Niederschlag aus; diese wurde von Baryt befreit und mit einer Lösung von essigsaurem Kupfer versetzt. Es bildete sich ein ganz geringer Niederschlag, der nicht weiter untersucht wurde. Nun könnten aber die vielfachen Operationen 
den Verlust dieser Säure verursachen. Die Ausbeute aus den Phosphorwolframsäureniederschlägen war folgende:

Glykokoll $17 \mathrm{~g}$

Alanin 3*

Oxyprolin 15 "

Glutaminsäure 6 »

$\alpha$-Prolin.

Die alkohollöslichen Kupfersalze wurden von Kupfer befreit und mit den alkoholisch-ätherischen Auszügen aus den anderen Fraktionen vereinigt. Sie wurden so weit wie möglich eingedampft und wieder mit einem Gemisch von Alkohol und Äther extrahiert. Der alkoholisch-ätherische Auszug wurde ân der Luft verdunstet. Dabei schied sich ein Niederschlag aus, der nach einmaligem Umkrystallisieren' aus absolutem Alkohol alle Eigenschaften des $\alpha$-Prolins besaß. Die Zusammensetzung war die folgende:

$0,1087 \mathrm{~g}$ der Substanz gaben bei der Verbrennung $0,2077 \mathbf{g ~ C O}_{\mathbf{z}}$ und 0,0810 g $_{2} \mathrm{O}$.
Für $\mathrm{C}_{5} \mathrm{H}_{9} \mathrm{NO}_{2}$
Berechnet:
$\mathrm{C}=52,17 \%$
$\mathrm{H}=7,82 \%$
Gefunden:
$52,10 \%$
$8,28 \%$

Die Mutterlauge vom aktiven $\alpha$-Prolin, welche $1,5 \mathrm{~g}$ Stickstoff enthielt, wurde mit Barythydrat racemisiert und in das Kupfersalz des inaktiven $\alpha$-Prolins übergeführt.

Die Ausbeute an dieser Substanz betrug 6,0 g. Über die Gewinnung von Oxyprolin und von $\alpha$-Prolin ohne Anwendung von Phosphorwolframsäure.

Das Vorkommen von Oxyprolin in fast allen Phosphorwolframsäureniederschlägen, die nach der Entfernung von Leucin und Glykokoll erhalten wurden, und die verhältnismäßig leichte Isolierung der Substanz haben zu dem Versuch, sie auch ohne Anwendung von Phosphorwolframsäure darzustellen; Veranlassung gegeben.

$300 \mathrm{~g}$ Gelatine sind bei diesem Experimente zur Anwendung gekommen. Die Behandlung war dieselbe wie im 
vorigen Versuche. Nur wurde die Trennung mittels Pikrinsäure vorgenommen, nachdem alles Leucin entfernt war. Der Pikrinsäureniederschlag und das Filtrat wurden beide von der Pikrinsäure befreit und die Aminosäurelösungen in die Kupfersalze übergeführt, die Lösungen derselben wurden soweit wie möglich konzentriert und mit absolutem Alkohol ausgekocht. Man erhielt auf diesem Wege alkohollösliche und unlösliche Fraktionen der Kupfersalze.

Die beiden alkohollöslichen Teile wurden vereinigt, von Kupfer befreit, eingedampft, bis zu ganz kleinem Volumen in wenig absolutem Alkohol aufgenommen, mit Äther bis zu starker Opalescenz versetzt und stehen gelassen. Es bildete sich ein krystallinischer Niederschlag und aus der Mutterlauge wurde nach weiterer Zugabe von Äther eine zweite Krystallisation erhalten. Beim Umkrystallisieren dieser Niederschläge schieden sich die typischen Krystalle des Oxyprolins aus. Die Ausbeute betrug $9,0 \mathrm{~g}$.

Die alkoholunlöslichen Fraktionen enthalten noch mehr Oxyprolin. Um dieses zu isolieren, wurden erst die in Wasser und in verdünntem Alkohol unlöslichen Teile entfernt und nun die in verdünntem Alkohol lösliche Fraktion weiter verarbeitet. Sie wurde von Kupfer befreit, bis zu kleinem Volumen eingedampft und der Rückstand mit Alkohol übergossen und bei Gefriertemperatur stehen gelassen. Es bildete sich eine klebrige Masse, die bei weiterem Stehen teilweise krystallisierte. Das Filtrieren erwies sich als ziemlich schwierig, doch beim Umkrystallisieren erhielt man typisches Oxyprolin. Die Mutterlauge von diesem Niederschlage wurde wieder eingedampft und mit Methylalkohol extrahiert; der Niederschlag bildete beim Umkrystallisieren prachtvolle Oxyprolinkrystalle. Die Ausbeute an Oxyprolin aus den in verdünntem Alkohol löslichen Kupfersalzen betrug $10,2 \mathrm{~g}$.

Es wird also in Zukunft ratsam sein, zur Gewinnung des Oxyprolins die Aminosäuren in die Kupfersalze überzuführen, die in Wasser und in verdünntem Alkohol unlöslichen Fraktionen $\mathrm{zu}$ entfernen und die in verdünntem Alkohol löslichen Teile zur Gewinnung des Oxyprolins zu verarbeiten. 
Die Mutterlaugen von Oxyprolin sind gut geeignet zur Gewinnung des $\alpha$-Prolins. Dazu werden sie eingedampft und mit absolutem Alkohol extrahiert. Die Operation wird noch einmal wiederholt. Der zweite Auszug enthielt 2,5 g Stickstoff. Die Fraktion wurde in üblicher Weise racemisiert, die baryumfreie Lösung eingedampft und der Rückstand mit absolutem Alkohol extrahiert - er löste sich fast vollständig. Dieser Auszug wurde zur Darstellung des Kupfersalzes des inaktiven $\alpha$-Prolins benutzt. Es gelang, 9,0 g des lufttrockenen Salzes zu gewinnen.

Beim Racemisieren der nach Fischers Verfahren erhaltenen Prolinfraktion ist es Abderhalden gelungen, aus $1000 \mathrm{~g}$ Gelatine $19 \mathrm{~g}$ der Substanz zu gewinnen.

Die Ausbeute an verschiedenen Aminosäuren in der vorliegenden Arbeit war die folgende:

\begin{tabular}{lccc} 
& Gewicht & Gewicht des N & $\begin{array}{c}\text { Prozent des } \\
\text { Gesamtgewichtes }\end{array}$ \\
Glykokoll & $\mathbf{g}$ & $\mathbf{g}$ & 19,25 \\
Alanin & $\mathbf{7 7}$ & 13,86 & 3,00 \\
Leucin & 12 & 1,80 & 6,75 \\
a-Prolin & 27 & 3,24 & 6,25 \\
Oxyprolin & 25 & 3,20 & 6,4 \\
Phenylalanin & 25,6 & 2,73 & - \\
Asparaginsäure & vermi & - & - \\
Glutaminsäure & 7 & - & 1,75 \\
\cline { 2 - 4 } & 173,6 & 25,53 & $-13,40$
\end{tabular}

Im Filtrate von der Fällung mittels $10 \%$ iger Phosphorwolframsäure, also in der Aminosäurefraktion, waren $50 \mathrm{~g}$ Stickstoff enthalten, also die Hälfte des Stickstoffs wurde vermißt. 